\title{
THE POWER PROBLEM FOR GROUPS WITH ONE DEFINING RELATOR
}

\author{
JAMES MCCOOL
}

Abstract. It is proved that if $G$ is a group with one defining relator, then the generalized word problem is solvable for every cyclic subgroup of $G$. This result enables the solution of the word problem for groups with one defining relator to be extended to a wider class of groups.

1. Introduction. Let $G$ be a group given by a presentation $\langle S ; D\rangle$, and let $H$ be a subgroup of $G$, generated by a set $A$ of words in the elements of $S$. The generalized word problem (GWP) for $H$ in $G$ is the algorithmic problem of deciding whether or not an arbitrary word $U \in G$ is an element of $H$. If the GWP is solvable for every cyclic subgroup of $G$, then $G$ is said to have solvable power problem. The object of this note is to prove the following

TheOREM. Let $G$ have presentation $\langle t, b, c, \cdots ; R(t, b, c, \cdots)\rangle$. Then the power problem is solvable for $G$.

The order problem for a group $G=\langle S ; D\rangle$ is the algorithmic problem of deciding the order of an arbitrary word $U \in G$. A solution to this problem for groups with a single defining relator is given by the results of $\$ 4.4$ of [2]. It follows that Theorem 6 of [3] '(see also Theorem 5 of [1]) can be applied to extend the solution of the word problem for groups with a single defining relator to a wider class of groups. As a simple example of this, we have the following generalization of Corollary 4.14.1 of [2].

COROLlary. Let $G_{1}$ and $G_{2}$ have presentations

$$
\left\langle a_{1}, \cdots, a_{m} ; R\left(a_{1}, \cdots, a_{m}\right)\right\rangle \text { and }\left\langle b_{1}, \cdots, b_{n} ; S\left(b_{1}, \cdots, b_{n}\right)\right\rangle
$$

respectively, and let $U\left(a_{1}, \cdots, a_{m}\right), V\left(b_{1}, \cdots, b_{n}\right)$ be elements of $G_{1}$, $G_{2}$ respectively, such that the orders of these elements are equal. Then the order problem and the power problem are solvable for the group

$$
G=\left\langle a_{1}, \cdots, a_{m}, b_{1}, \cdots, b_{n} ; R, S, U=V\right\rangle .
$$

2. Proof of the theorem. We shall make use of the following generalization of Lemma 2 of [1]. The proof requires only trivial

Received by the editors July 16, 1970.

AMS 1969 subject classifications. Primary 2010.

Key words and phrases. Group presentation, one defining relator, generalized word problem, cyclic subgroups. 
modification of that of Theorem 5 of [1], and so is omitted.

Lemma. Let the groups $G_{0}$ and $G_{1}$ have presentations

$$
\left\langle a_{1}, a_{2}, \cdots, b_{1}, b_{2}, \cdots ; R_{1}, R_{2}, \cdots\right\rangle
$$

and

$$
\left\langle a_{1}, a_{2}, \cdots, c_{1}, c_{2}, \cdots ; S_{1}, S_{2}, \cdots\right\rangle,
$$

respectively, and suppose that the following conditions are satisfied:

(a) The power problem is solvable for $G_{0}$ and $G_{1}$.

(b) The subgroups $H_{i}$ of $G_{i}(i=0,1)$ generated by the corresponding elements $a_{1}, a_{2}, \cdots$ are isomorphic under the identity mapping.

(c) The GWP for $H_{i}$ in $G_{i}(i=0,1)$ is solvable.

Then the power problem is solvable for

$$
G=\left\langle a_{1}, a_{2}, \cdots, b_{1}, b_{2}, \cdots, c_{1}, c_{2}, \cdots ; R_{1}, R_{2}, \cdots, S_{1}, S_{2}, \cdots\right\rangle,
$$

the free product of $G_{0}$ and $G_{1}$ amalgamating $H_{0}$ with $H_{1}$.

We prove the theorem by induction on the length of the relator $R$. The method of proof is the one used repeatedly in $\$ 4.4$ of [2], so we have omitted many of the details.

We can suppose that $R$ as written is cyclically reduced. If $R$ involves only one generator, it is easy to see that the result holds. Thus we assume that $R$ involves at least two generators, say $t$ and $b$, and that the result holds for all groups with one defining relator of length less than that of $R$.

Case 1. $R$ has zero exponent sum on some generator; say $\sigma_{t}(R)=0$.

Let $U$ and $V$ be elements of $G$. We show that we can decide whether or not there exists an integer $n$ such that $U=V^{n}$. Using the solution of the order problem for $G$, it is easy to dispose of the case when either $U$ or $V$ has finite order; thus we assume that $U$ and $V$ have infinite order.

Now if $U=V^{n}$ for some integer $n$ (which must be nonzero), then $U V^{-n} \in N$, the normal subgroup of $G$ generated by $b, c, \cdots$, and so $\sigma_{t}\left(U V^{-n}\right)=0$. Thus, putting $\lambda=\sigma_{t}(U)$ and $\eta=\sigma_{t}(V)$, we must have $\lambda-n \eta=0$.

Suppose that $\lambda \neq 0$. Then, if $U=V^{n}$, we have $\eta$ divides $\lambda$ and $n$ $=\lambda / \eta$. Thus in this case there is at most one value of $n$ to test.

Thus we can assume that $\lambda=0$. If $\eta \neq 0$, then, since $n \neq 0$, we cannot have $\lambda-n \eta=0$. Thus we can assume also that $\eta=0$. In other words, we can assume that both $U$ and $V$ are elements of $N$.

We now show that $N$ has solvable power problem. We have 


$$
N=\left\langle\cdots, b_{-1}, b_{0}, b_{1}, \cdots, c_{-1}, c_{0}, c_{1}, \cdots ; \cdots, P_{-1}, P_{0}, P_{1}, \cdots\right\rangle,
$$

where, for $k=0, \pm 1, \pm 2, \cdots, b_{k}, c_{k}, \cdots$ denote the elements $t^{k} b t^{-k}, t^{k} c t^{k}, \cdots$ respectively, and $P_{k}$ is the element $t^{k} R t^{-k}$ rewritten in terms of these generators.

Now the subgroup $N_{i}$ of $N$ generated by

$$
\cdots, c_{-1}, c_{0}, c_{1}, \cdots, b_{\mu+i}, \cdots, b_{M+i},
$$

where $\mu$ is the minimum subscript on $b$ involved in $P_{0}$, and $M$ is the maximum subscript on $b$ involved in $P_{0}$, has presentation

$$
N_{i}=\left\langle\cdots, c_{-1}, c_{0}, c_{1}, \cdots, b_{\mu+i}, \cdots, b_{M+i} ; P_{i}\right\rangle .
$$

Thus $N_{i}$ is a group with one defining relator $P_{i}$. Moreover, the length of $P_{i}$ is less than that of $R$, so that, by the inductive hypothesis, the power problem is solvable for $N_{i}$.

Now, as in the proof of Theorem 4.14 of [2], we can describe $N$ as the union of a chain of groups

$$
Q_{1}=N_{0} \subset Q_{2} \subset \cdots \subset Q_{s} \subset Q_{s+1} \subset \cdots
$$

We prove, by induction on $s$, that each $Q_{s}$ has solvable power problem; it will then follow that $N$ has solvable power problem.

We suppose that the power problem is solvable for $Q_{s}$. Now $Q_{s+1}$ is the free product of $Q_{s}$ and some $N_{p}$, with the subgroup $K$ of $N_{p}$ generated by all the generators of $N_{p}$ except some $b_{k}$ amalgamated under the identity mapping. Denote this set of generators by $A$. Then $A$ is a subset of the generators of some $N_{q}$ whose generators are among the generators of $Q_{s}$. Now the GWP for $K$ in $N_{p}$ is solvable, as is the GWP for $K$ in $N_{q}$, by Theorem 4.14 of [2]; moreover, in the proof of that theorem, it is shown that if the generators of $N_{q}$ are among the generators of $Q_{s}$, then the GWP for $N_{q}$ in $Q_{s}$ is solvable. It follows that the GWP for $K$ in $Q_{s}$ is solvable. Thus we can apply the lemma, to deduce that the power problem is solvable for $Q_{*+1}$. Hence the power problem is solvable for each $Q_{s}$, and so is also solvable for $N$.

Case 2. All the generators in $R$ have nonzero exponent sums.

Put $\alpha=\sigma_{t}(R)$ and $\beta=\sigma_{b}(R)$. Then $G$ is (effectively) embedded in the group $G_{1}$ with presentation

$$
G_{1}=\left\langle x, y, c, \cdots ; R\left(y x^{-\beta}, x^{\alpha}, c, \cdots\right)\right\rangle .
$$

Thus the power problem is solvable for $G$ if it is solvable for $G_{1}$. But the exponent sum of $x$ in $R\left(y x^{-\beta}, x^{\alpha}, c, \cdots\right)$ is zero, and when this relator is rewritten in terms of the (usual) generators of the normal 
subgroup of $G_{1}$ generated by $y, c, \cdots$, the relator obtained has length less that that of $R(t, b, c, \cdots)$. Thus, using the same argument as in Case 1, we see that the power problem is solvable for $G_{1}$. This proves the theorem.

\section{REFERENCES}

1. S. Lipschutz, An extension of Greendlinger's results on the word problem, Proc. Amer. Math. Soc. 15 (1964), 37-43. MR $28 \# 4018$.

2. W. Magnus, A. Karrass and D. Solitar, Combinatorial group theory; Presentation of groups in terms of generators and relations, Pure and Appl. Math., vol. 13, Interscience, New York, 1966. MR 34 \#7617.

3. J. McCool, The order problem and the power problem in free product sixth-groups, Glasgow Math. J. 10 (1969), 1-9. MR 39 \#2852.

University of Toronto, Toronto, Canada 